\title{
Quantum dynamics of impurities coupled to a Fermi sea
}

\author{
Meera M. Parish and Jesper Levinsen \\ School of Physics and Astronomy, Monash University, Victoria 3800, Australia.
}

(Dated: June 3, 2021)

\begin{abstract}
We consider the dynamics of an impurity atom immersed in an ideal Fermi gas at zero temperature. We focus on the coherent quantum evolution of the impurity following a quench to strong impurity-fermion interactions, where the interactions are assumed to be short range like in coldatom experiments. To approximately model the many-body time evolution, we use a truncated basis method, where at most two particle-hole excitations of the Fermi sea are included. When the system is initially non-interacting, we show that our method exactly captures the short-time dynamics following the quench, and we find that the overlap between initial and final states displays a universal non-analytic dependence on time in this limit. We further demonstrate how our method can be used to compute the impurity spectral function, as well as describe many-body phenomena involving coupled impurity spin states, such as Rabi oscillations in a medium or highly engineered quantum quenches.
\end{abstract}

\section{INTRODUCTION}

The coherent evolution of quantum many-body systems out of equilibrium defines a new frontier in current research, and is of fundamental importance to a number of fields, ranging from neutron stars to electronic devices. In fermionic systems, the investigation of dynamics at the relevant time scale - the Fermi time $\tau_{F}=\hbar / \varepsilon_{F}$, with $\varepsilon_{F}$ the Fermi energy - has recently become available due to advances in the field of ultracold atoms. The coldatom system possesses a number of unique advantages over its solid-state counterparts [1]. Most notably, the parameters of the governing models are precisely known and can be rapidly changed; the cold-atom system is well isolated; and the real time observation of coherent manybody dynamics is experimentally accessible. The Fermi time is typically in the microsecond range, in stark contrast to the solid-state scenario where $\tau_{F}$ is shorter by about 10 orders of magnitude due to the much lighter particles and higher densities. The possibility of probing the coherent dynamics of ultracold Fermi gases has stimulated a large theoretical effort to understand interaction quenches in the crossover from Bardeen-CooperSchrieffer (BCS) type superfluidity to a Bose-Einstein condensate (BEC) of tightly bound pairs [2-4].

A particularly clean realization of coherent dynamics on the Fermi time scale is afforded by population imbalanced Fermi gases [5-13]. Here, it is possible to investigate the dynamical response of a many-fermion system to the sudden introduction of an impurity. This response plays a central role in important phenomena such as the orthogonality catastrophe [14]. A recent experiment employed Ramsey interferometry on heavy ${ }^{40} \mathrm{~K}$ impurity atoms immersed in a ${ }^{6} \mathrm{Li}$ Fermi sea, revealing both the real-time formation of impurity quasiparticles as well as the interference between attractive and repulsive polaron branches [15]. The Ramsey protocol provides a direct measure of the time-dependent overlap function at time $t[16,17]$

$$
S(t)=\left\langle\psi_{0}\left|e^{i \hat{H}_{0} t} e^{-i \hat{H}_{\mathrm{int}} t}\right| \psi_{0}\right\rangle,
$$

where $\left|\psi_{0}\right\rangle$ is the initial non-interacting state of the total system, $t=0$ defines the point where the impurity starts interacting with the Fermi sea, and $\hat{H}_{0}$ and $\hat{H}_{\text {int }}$ correspond to the Hamiltonians in the absence and presence of interactions, respectively. As such, Ramsey interferometry provides detailed information on the time evolution of the impurity wave function. Throughout this article, we focus on the purely quantum evolution at zero temperature, and we work in units where $\hbar$, the Boltzmann constant $k_{B}$ and the volume are all set to 1 .

The dynamical response of a strongly interacting quantum many-body system is a challenge to determine theoretically since the interactions cannot be treated perturbatively. Here, we present a theoretical approach to determine the coherent impurity dynamics based on truncating the Hilbert space of impurity wave functions at a fixed number of particle-hole excitations of the Fermi sea. As we demonstrate, this truncated basis method (TBM) allows us to capture the Ramsey response exactly at times $t \ll \tau_{F}$, where two-body correlations dominate. We also consider the challenging scenario of an infinitely heavy (static) impurity, where one has the orthogonality catastrophe [14], and the overlap in Eq. (1) exhibits a power-law decay at long times arising from the multiple low-energy excitations of the Fermi sea. In this case, we show that the TBM provides results that are essentially exact to several $\tau_{F}$. Hence, the TBM likely provides a near exact solution up to several $\tau_{F}$ for coherent impurity dynamics in the strongly interacting regime, even when the impurity mass is finite. Furthermore, we argue that the TBM also captures the long-time Ramsey response in cases where the attractive polaron $[18,19]$ is well-defined.

A key result of the present work is the exact short-time evolution of the Ramsey response, which is dominated by two-body physics for $t \ll \tau_{F}$. In the case where the shortrange interaction of the $|\uparrow\rangle$ impurity with the Fermi sea is described by a single parameter, the scattering length $a$, the Ramsey response takes the form

$$
S(t) \simeq 1-\frac{8 e^{-i \pi / 4}\left(m / m_{r}\right)^{3 / 2}}{9 \pi^{3 / 2}}\left(\frac{t}{\tau_{F}}\right)^{\frac{3}{2}} .
$$


Here, $m\left(m_{\mathrm{im}}\right)$ is the mass of a majority (impurity) particle, and $m_{r}=m m_{\mathrm{im}} /\left(m+m_{\mathrm{im}}\right)$ is the reduced mass. Note that Eq. (2) does not depend on the scattering length and, furthermore, it does not display the short-time behavior expected from a simple expansion of the time evolution operators, where we have $1-S(t) \propto$ $t^{2}$. Such a quadratic dependence on time is also expected for a Loschmidt echo [20], which is defined as a time-dependent wave function overlap similar to Eq. (1) and which yields information about an imperfect timereversal procedure applied to a quantum system. Instead, the non-analytic behavior of $S(t)$ is a direct consequence of the renormalization of the contact interactions. For resonances where the effective range $r_{\text {eff }}$ greatly exceeds the van der Waals range of the interatomic interactions, $r_{\text {eff }}$ must be taken into account in the short time evolution. In this case, we find

$S(t) \simeq 1-\frac{\left(m / m_{r}\right)^{2}}{3 \pi k_{F} R^{*}}\left(\frac{t}{\tau_{F}}\right)^{2}+\frac{16 e^{i \pi / 4}\left(m / m_{r}\right)^{5 / 2}}{45 \pi^{3 / 2}\left(k_{F} R^{*}\right)^{2}}\left(\frac{t}{\tau_{F}}\right)^{\frac{5}{2}}$,

where $k_{F}=\sqrt{2 m \varepsilon_{F}}$ is the Fermi momentum and we define the positive range parameter $R^{*}=-r_{\text {eff }} / 2$. Again, the Ramsey response is independent of scattering length, and while the leading order contribution has the expected form of a Loschmidt echo, the next order correction is non-analytic.

The TBM provides us with a framework in which impurity dynamics can be explored systematically. To illustrate this point, we apply it to two scenarios of coherent impurity dynamics beyond the Ramsey response: Rabi oscillations between quasiparticle branches, and the dynamical preparation of strongly interacting quantum states. We also show how the TBM allows the straightforward calculation of the impurity spectral function.

The paper is organized as follows. In Sec. II, we describe the model Hamiltonian, while in Sec. III we outline the truncated basis method. In Sec. IV we present our results for the Ramsey response, including the analytic short and long-time behavior, as well as for the impurity spectral function. Sections V and VI discuss, respectively, Rabi oscillations and how the initial quantum state can be modified. Section VII then examines the role played by multiple particle-hole excitations, focussing for simplicity on a static impurity. We conclude in Sec. VIII.

\section{MODEL}

In the following, we consider the dynamics of a single impurity immersed in a Fermi gas. For this purpose, it is convenient to consider two impurity spin states, $\sigma=\downarrow, \uparrow$, of which one $(\uparrow)$ is strongly interacting with the Fermi sea, while the other $(\downarrow)$ is non-interacting. To model interactions, we employ a two-channel Hamiltonian. Restricting ourselves at first to the part of the Hamilto- nian describing the interacting $\uparrow$ impurity state and the medium, we have

$$
\begin{aligned}
\hat{H}_{\mathrm{int}} & =\sum_{\mathbf{k}} \epsilon_{\mathbf{k}, \mathrm{im}} \hat{c}_{\mathbf{k} \uparrow}^{\dagger} \hat{c}_{\mathbf{k} \uparrow}+\sum_{\mathbf{k}} \epsilon_{\mathbf{k}} \hat{f}_{\mathbf{k}}^{\dagger} \hat{f}_{\mathbf{k}}+\sum_{\mathbf{k}}\left[\epsilon_{\mathbf{k}, M}+\nu\right] \hat{d}_{\mathbf{k}}^{\dagger} \hat{d}_{\mathbf{k}} \\
& +g \sum_{\mathbf{k}, \mathbf{q}}\left(\hat{d}_{\mathbf{q}}^{\dagger} \hat{f}_{\mathbf{q} / 2+\mathbf{k}} \hat{c}_{\mathbf{q} / 2-\mathbf{k}, \uparrow}+\hat{c}_{\mathbf{q} / 2-\mathbf{k}, \uparrow}^{\dagger} \hat{f}_{\mathbf{q} / 2+\mathbf{k}}^{\dagger} \hat{d}_{\mathbf{q}}\right) .
\end{aligned}
$$

The first line of Eq. (4) corresponds to the noninteracting Hamiltonian $\hat{H}_{0}$, where $\hat{c}_{\mathbf{k} \sigma}^{\dagger}\left(\hat{c}_{\mathbf{k} \sigma}\right)$ creates (annihilates) an impurity particle with momentum $\mathbf{k}$, spin $\sigma$, mass $m_{\mathrm{im}}$, and single particle energy $\epsilon_{\mathbf{k}, \mathrm{im}}=\frac{k^{2}}{2 m_{\mathrm{im}}}$. Likewise, the operators $\hat{f}_{\mathbf{k}}^{\dagger}$ and $\hat{f}_{\mathbf{k}}$ respectively create and annihilate a majority fermion with momentum $\mathbf{k}$, mass $m$, and single particle energy $\epsilon_{\mathbf{k}}=\frac{k^{2}}{2 m}$. The spin- $\uparrow$ impurity interacts with the fermions by forming a closed channel molecule described by the creation and annihilation operators $\hat{d}_{\mathbf{k}}^{\dagger}$ and $\hat{d}_{\mathbf{k}}$ with momentum $\mathbf{k}$, single-particle energy $\epsilon_{\mathbf{k}, \mathrm{M}}=\frac{k^{2}}{2 M}$, and mass $M=m+m_{\mathrm{im}}$. The detuning of this closed channel molecule from the impurity-fermion scattering threshold is denoted $\nu$. The interaction second line of Eq. (4) - has a coupling strength $g$ for relative momenta with magnitude $|\mathbf{k}|<\Lambda$, where $\Lambda$ is a UV cut-off.

Using standard techniques (see, e.g., Ref. [21]), we relate the bare interaction parameters $g, \Lambda$, and $\nu$ to renormalized quantities by calculating the low-energy spin- $\uparrow$ impurity-fermion scattering amplitude at a relative momentum $\mathbf{k}$ within the model (4). We then compare the resulting expression with the standard low-energy expansion of the scattering amplitude

$$
f(\mathbf{k})=-\frac{1}{a^{-1}-\frac{1}{2} r_{\mathrm{eff}} k^{2}+i k},
$$

where $a$ and $r_{\text {eff }}$ are the scattering length and effective range, respectively. This procedure yields the scattering length $a$ through

$$
\frac{m_{r}}{2 \pi a}=-\frac{\nu}{g^{2}}+\sum_{\mathbf{k}}^{\Lambda} \frac{1}{\epsilon_{\mathbf{k}}+\epsilon_{\mathbf{k}, \mathrm{im}}},
$$

In particular, we see how the model allows us to tune the scattering length to resonance, $1 / a=0$. For resonances where $\left|r_{\text {eff }}\right|$ greatly exceeds the range of the bare interaction, $r_{\text {eff }}$ is negative and we instead define the range parameter [22]

$$
R^{*}=-r_{\mathrm{eff}} / 2=\frac{\pi}{m_{r}^{2} g^{2}} .
$$

We emphasize that the model (4) reduces to the commonly used single-channel model with $R^{*}=0$ by taking $g, \nu \rightarrow \infty$ in such a way that $\nu / g^{2}=m_{r} \Lambda / \pi^{2}-m_{r} /(2 \pi a)$.

The presence of the auxiliary $\downarrow$ state enables one to probe impurity dynamics starting from a non-interacting 
state. For instance, interactions between the impurity and the medium can suddenly be switched on by using a radiofrequency (rf) pulse which couples the $\downarrow$ and $\uparrow$ impurity states. The total Hamiltonian is then $\hat{H}=\hat{H}_{\text {int }}+\hat{H}_{\text {aux }}$ with

$$
\begin{aligned}
\hat{H}_{\mathrm{aux}}= & \sum_{\mathbf{k}}\left(\epsilon_{\mathbf{k}, \mathrm{im}}+\delta\right) \hat{c}_{\mathbf{k} \downarrow}^{\dagger} \hat{c}_{\mathbf{k} \downarrow} \\
& +\frac{\Omega_{0}}{2 i} \sum_{\mathbf{k}}\left(e^{i \varphi} \hat{c}_{\mathbf{k} \downarrow}^{\dagger} \hat{c}_{\mathbf{k} \uparrow}-e^{-i \varphi} \hat{c}_{\mathbf{k} \uparrow}^{\dagger} \hat{c}_{\mathbf{k} \downarrow}\right) .
\end{aligned}
$$

Here, $\Omega_{0}$ and $\varphi$ are the Rabi frequency and phase of the rf pulse, respectively, and $\delta \equiv \omega-\omega_{0}$ is the detuning of the rf pulse with frequency $\omega$ from the bare $\downarrow-\uparrow$ transition frequency, $\omega_{0}$. Note that we have applied the rotating wave approximation, assuming $\left|\delta /\left(\omega+\omega_{0}\right)\right| \ll 1$. For the remainder of this manuscript, we set $\omega_{0}$ to zero.

\section{TRUNCATED BASIS METHOD}

To formulate our approach to the many-body dynamics, we start from the time-dependent variational principle, which is applicable to any many-body system and is not limited to impurity dynamics. Here, we wish to determine the time evolution of an approximate variational wave function $\psi(t)$ that best describes that of the actual system. To this end, we consider the action of the operator $\hat{\epsilon}=i \partial_{t}-\mathcal{H}$, where $\mathcal{H}$ is the Hamiltonian that governs the dynamics of the system. Clearly, if $\psi$ were the exact wave function, then $\hat{\epsilon} \psi=0$. More generally, if we know $\psi(t)$ at time $t$ and we wish to approximately determine $\psi(t+\delta t)$, we must minimise the "error" quantity [23]

$$
\Delta=\int|\hat{\epsilon} \psi(t)|^{2} d V
$$

with respect to the unknown function $\partial_{t} \psi$, where the above integral is over all space. There are also other formulations of the time-dependent variational principle that give equivalent results [24].

In what follows, we will consider wave functions of the form: $|\psi\rangle=\sum_{j} \gamma_{j}|j\rangle$, where $\{|j\rangle\}$ represents a subset of a complete orthonormal set of states. Within this truncated basis, Eq. (9) becomes

$$
\begin{aligned}
\Delta= & i \sum_{j}\left(\dot{\gamma}_{j}^{*}\langle j|\mathcal{H}| \psi\rangle-\dot{\gamma}_{j}\langle\psi|\mathcal{H}| j\rangle\right) \\
& +\sum_{j, l}\langle j \mid l\rangle \dot{\gamma}_{j}^{*} \dot{\gamma}_{l}+\left\langle\psi\left|\mathcal{H}^{2}\right| \psi\right\rangle,
\end{aligned}
$$

where $\dot{\gamma}_{j}=\frac{d \gamma_{j}}{d t}$. Imposing the condition $\frac{\partial \Delta}{\partial \dot{\gamma}_{j}^{*}}=0$ then gives

$$
\langle j|\hat{\epsilon}| \psi\rangle=0 \text {. }
$$

Exploiting the orthonormality of the basis states, $\langle j \mid l\rangle=$ $\delta_{j l}$, finally yields the equations of motion:

$$
i \frac{d \gamma_{j}}{d t}=\sum_{l}\langle j|\mathcal{H}| l\rangle \gamma_{l} \equiv \sum_{l} \mathcal{H}_{j l} \gamma_{l} .
$$

Note that the norm of the wave function is preserved in this case since we have $\langle\psi|\hat{\epsilon}| \psi\rangle=0$, i.e.:

$$
\frac{d}{d t}\langle\psi \mid \psi\rangle=i(\langle\psi|\mathcal{H}| \psi\rangle-\langle\psi|\mathcal{H}| \psi\rangle)=0 .
$$

Equivalently, we can see this from the fact that the time evolution operator within this subspace is unitary.

\section{A. General solution for a quench}

To determine the approximate time evolution of a system, one must in general solve the set of coupled differential equations (12) directly. However, the situation simplifies when the system evolves under a time-independent Hamiltonian. This includes the scenario where there is an abrupt change in the parameters of the Hamiltonian at some time $t_{0}$, i.e., the system undergoes a quench, which is the focus of this paper.

In this case, one proceeds by solving for the eigenstates of the projected Hamiltonian $\mathcal{H}_{j l}$, using the equations for the energy $E$ :

$$
E \gamma_{j}=\sum_{l} \mathcal{H}_{j l} \gamma_{l}
$$

and then expanding the system's wave function $|\psi(t)\rangle$ in terms of these eigenstates. To be concrete, suppose we start from some initial state $|\psi(0)\rangle$, and we instantaneously "turn on" the effect of the Hamiltonian $\mathcal{H}$ at time $t=0$. The resulting wave function is

$$
|\psi(t)\rangle=e^{-i \mathcal{H} t}|\psi(0)\rangle \simeq \sum_{n}\left\langle\phi_{n} \mid \psi(0)\right\rangle e^{-i E_{n} t}\left|\phi_{n}\right\rangle,
$$

where $\left|\phi_{n}\right\rangle$ are the eigenstates within the $\{|j\rangle\}$ subspace, and $E_{n}$ are the corresponding eigenenergies. Note that this implicitly assumes that the eigenstates are orthogonal, but this is guaranteed from the fact that the Hamiltonian is Hermitian in this subspace.

\section{B. Impurity wave function}

For the specific case of an impurity interacting with a Fermi medium, we consider wave functions of the form

$$
|\psi\rangle=\left|\psi_{\uparrow}\right\rangle+\left|\psi_{\downarrow}\right\rangle
$$

where

$\left|\psi_{\uparrow}\right\rangle=\left[\alpha_{0} \hat{c}_{\mathbf{0} \uparrow}^{\dagger}+\sum_{\mathbf{q}} \alpha_{\mathbf{q}} \hat{d}_{\mathbf{q}}^{\dagger} \hat{f}_{\mathbf{q}}+\sum_{\mathbf{k}, \mathbf{q}} \alpha_{\mathbf{k q}} \hat{c}_{\mathbf{q}-\mathbf{k} \uparrow}^{\dagger} \hat{f}_{\mathbf{k}}^{\dagger} \hat{f}_{\mathbf{q}}\right]|\mathrm{FS}\rangle$,

$\left|\psi_{\downarrow}\right\rangle=\left[\beta_{0} \hat{c}_{\mathbf{0} \downarrow}^{\dagger}+\sum_{\mathbf{k}, \mathbf{q}} \beta_{\mathbf{k q}} \hat{c}_{\mathbf{q}-\mathbf{k} \downarrow}^{\dagger} \hat{f}_{\mathbf{k}}^{\dagger} \hat{f}_{\mathbf{q}}\right]|\mathrm{FS}\rangle$ 
Here, $\quad|\mathrm{FS}\rangle=\prod_{|\mathbf{q}|<k_{F}} \hat{f}_{\mathbf{q}}^{\dagger}|0\rangle$ describes the noninteracting Fermi sea with energy $E_{0}=\sum_{|\mathbf{q}|<k_{F}} \epsilon_{\mathbf{q}}$. Thus, we require that $|\mathbf{k}|>k_{F}$ and $|\mathbf{q}|<k_{F}$ for particle and hole excitations, respectively, in the impurity wave functions. We have implicitly assumed the impurity to have zero momentum, which is a good approximation in the limit of a small impurity density and zero temperature. The wave functions illustrate how the impurity can excite particles out of the Fermi sea leaving holes behind, and in both spin channels we truncate the wave function at one particle-hole excitation. The difference between $\left|\psi_{\uparrow}\right\rangle$ and $\left|\psi_{\downarrow}\right\rangle$ arises from the fact that the interacting spin $\uparrow$ impurity can bind a majority fermion to form a closed-channel molecule. Note that similar wave functions have been used to describe the equilibrium properties of an impurity in a Fermi gas: For the ground state, this includes the attractive quasiparticle $[18,25,26]$ and the transition to a dressed dimer state [27-29], as well as the transition to a trimer ground state [30]. Such wave functions have also been used to describe the metastable upper branch, i.e., the repulsive polaron [31].

When diagonalizing the Hamiltonian within the truncated basis spanned by states of the form (16), we note that the interaction part of the Hamiltonian, Eq. (4), still contains both the bare coupling $g$ and the detuning $\nu$. While $g$ is related to $R^{*}$ via Eq. (7), it is not possible to immediately trade the detuning for the renormalized interaction parameter, the scattering length, as the momentum cut-off cannot be taken to infinity. Thus, in practice we apply the following procedure: For a given $a$ and $R^{*}$, we first choose a momentum cut-off $\Lambda$ and adjust $\nu$ according to Eq. (6) to obtain the desired scattering length. Next, we increase the momentum grid to obtain convergent results at the chosen momentum cutoff, repeating this step for increasing cut-off to ensure convergence of the final result.

\section{COHERENT IMPURITY DYNAMICS FOLLOWING A QUENCH}

We now consider Ramsey interferometry and its relation to the impurity spectral function. To provide illustrations of the method in this section, we focus on equal masses $m_{\mathrm{im}}=m$. However, the approach can straightforwardly be extended to a mass imbalanced mixture [15].

\section{A. Dynamical response to an interaction quench}

We first consider the scenario where an impurity, initially in the non-interacting spin- $\downarrow$ state, is suddenly coupled to an interacting spin- $\uparrow$ state by an rf pulse. The many-body response to a rapidly introduced impurity into the Fermi gas can be probed by means of Ramsey interferometry [16, 17], as illustrated in Fig. 1(a): Following an initial $\pi / 2 \mathrm{rf}$ pulse, which creates a superposition of the impurity in $\downarrow$ and $\uparrow$ spin states, the system (a)

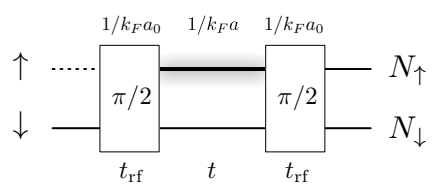

(b)

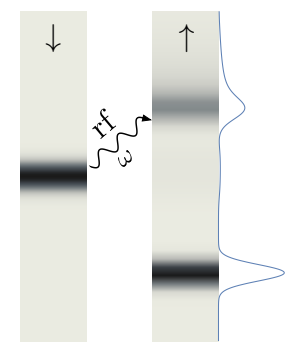

FIG. 1. Illustration of experimental procedures used to probe impurities in a Fermi gas. (a) In Ramsey interferometry, the impurities are initially in the non-interacting $|\downarrow\rangle$ state. At time $t=0$, they are in a superposition of $|\downarrow\rangle$ and $|\uparrow\rangle$ states following a $\pi / 2 \mathrm{rf}$ pulse of duration $t_{\mathrm{rf}}$. After a variable time $t$ and a second $\pi / 2 \mathrm{rf}$ pulse, the number of particles, $N_{\sigma}$, in the two impurity spin states is measured as a function of the phase of the second pulse. The interaction between the $\uparrow$ impurity state and the majority fermions is characterized by the interaction parameter $1 / k_{F} a_{0}$ during the rf pulses and $1 / k_{F} a$ during the evolution time. (b) In inverse rf spectroscopy, the spectral response of spin $\downarrow$ impurities to an rf pulse is measured as a function of frequency $\omega$ relative to the bare $\downarrow-\uparrow$ transition frequency (vertical axis).

evolves under the interacting Hamiltonian for a time $t$, after which a second $\pi / 2 \mathrm{rf}$ pulse is applied. For simplicity, in this section we consider a 'perfect quench' where no interactions take place during the rf pulses, and thus at time $t=0$ the impurities are in an equal superposition $\frac{1}{\sqrt{2}}(|\downarrow\rangle+|\uparrow\rangle)$. In this case, a measurement of the impurity population difference at the end of the Ramsey procedure yields [32]

$$
\frac{N_{\uparrow}-N_{\downarrow}}{N_{\uparrow}+N_{\downarrow}}=-\operatorname{Re}\left[e^{i \varphi_{\mathrm{rf}}} S(t)\right]+n_{d},
$$

where $\varphi_{\mathrm{rf}}$ is the phase of the second rf pulse with respect to the first, $n_{d}$ is the fraction of closed channel molecules at time $t$, and we have the overlap between interacting and non-interacting states

$$
S(t)=\left\langle\psi_{0}(t) \mid \psi_{\text {int }}(t)\right\rangle=e^{i E_{0} t}\left\langle\psi_{0}\left|e^{-i \hat{H}_{\mathrm{int}} t}\right| \psi_{0}\right\rangle,
$$

where $\left|\psi_{0}\right\rangle \equiv \hat{c}_{\mathbf{0} \uparrow}^{\dagger}|\mathrm{FS}\rangle$ and $\hat{H}_{0}\left|\psi_{0}\right\rangle=E_{0}\left|\psi_{0}\right\rangle$. By varying the relative phase $\varphi_{\mathrm{rf}}$, one can thus access both the amplitude and phase of $S(t)$.

According to the variational approach outlined in Sec. III A, we can determine an approximate Ramsey response $S(t)$ by diagonalizing the Hamiltonian within the subspace of wave functions of the form (16). In the perfect quench scenario, we only need to consider the decoupled spin-up part of the Hamiltonian, $\hat{H}_{\text {int }}$; thus we obtain the set of equations [33]:

$$
\begin{aligned}
\left(E-E_{0}\right) \alpha_{0} & =g \sum_{\mathbf{q}} \alpha_{\mathbf{q}}, \\
\left(E-E_{0}\right) \alpha_{\mathbf{q}} & =\left(\epsilon_{\mathbf{q}, \mathrm{M}}-\epsilon_{\mathbf{q}}+\nu\right) \alpha_{\mathbf{q}}+g \alpha_{0}+g \sum_{\mathbf{k}} \alpha_{\mathbf{k q}}, \\
\left(E-E_{0}\right) \alpha_{\mathbf{k q}} & =\left(\epsilon_{\mathbf{q}-\mathbf{k}, \mathrm{im}}+\epsilon_{\mathbf{k}}-\epsilon_{\mathbf{q}}\right) \alpha_{\mathbf{k q}}+g \alpha_{\mathbf{q}} .
\end{aligned}
$$



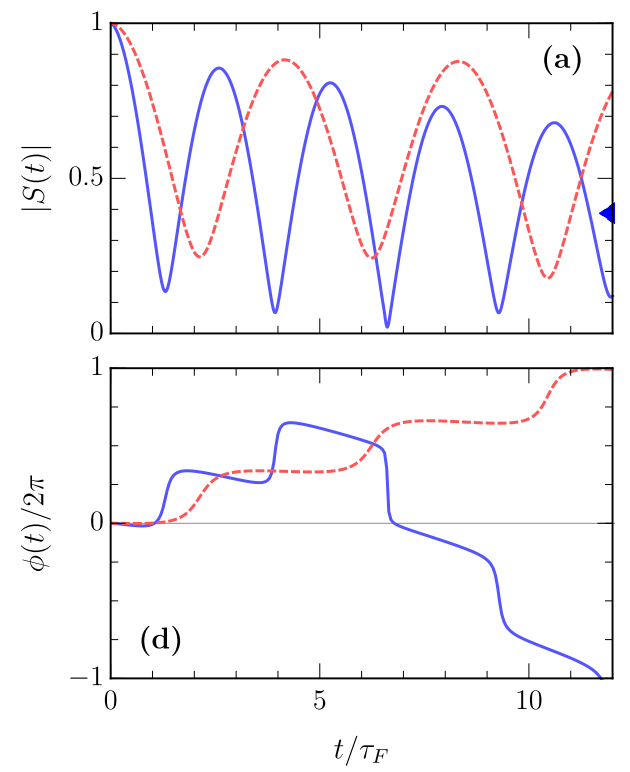
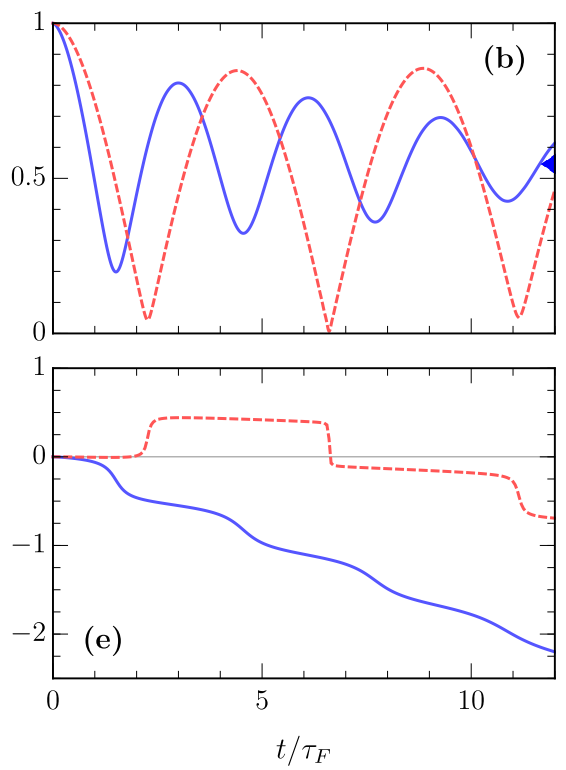
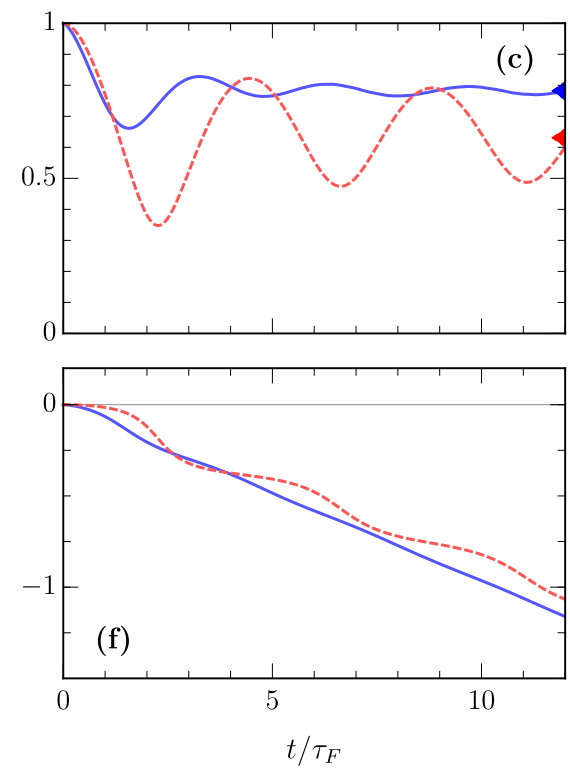

FIG. 2. The amplitude (top) and phase (bottom) of the Ramsey signal. We have taken the interaction parameter $1 / k_{F} a$ to be $(\mathrm{a}, \mathrm{d}) 0.73$, (b,e) 0.48 , and (c,f) 0 . The range parameter is $k_{F} R^{*}=0$ (solid) and $k_{F} R^{*}=1$ (dashed). The diamonds indicate the long-time limit of the Ramsey response in the cases where the attractive polaron is the ground state. The phase for $k_{F} R^{*}=0$ in (f) is well approximated by $\phi(t) \simeq E_{\text {att }} t$, where the attractive polaron energy $E_{\text {att }} \simeq-0.607 \varepsilon_{F}[18]$.

Solving these coupled equations yields the set of eigenstates $\left|\phi_{j}\right\rangle$ with corresponding energies $E_{j}$. We then obtain for the Ramsey response

$$
S(t) \simeq \sum_{j}\left|\left\langle\psi_{0} \mid \phi_{j}\right\rangle\right|^{2} e^{-i\left(E_{j}-E_{0}\right) t} .
$$

This expression has a natural interpretation. Up to a trivial phase, the contribution from the state $\left|\phi_{j}\right\rangle$ rotates at an angular frequency $E_{j}$, while the magnitude of the contribution is the squared overlap with the noninteracting ground state, i.e., the residue of $j$ 'th state: $Z_{j} \equiv\left|\left\langle\psi_{0} \mid \phi_{j}\right\rangle\right|^{2}$.

The time evolution of the impurity after an interaction quench is clearly intrinsically connected to the structure of its energy spectrum. As we discuss in more detail in Sec. IV B, the spectrum can contain well-defined quasiparticle states (the attractive and repulsive polarons) as well as a broad continuum of many-body states which have a vanishing overlap with the non-interacting system. The interference of these different states is, in general, expected to generate damped coherent oscillations in $|S(t)|$ as a function of time.

Figure 2 shows both the amplitude and the phase of $S(t) \equiv|S(t)| e^{-i \phi(t)}$ for different values of the interaction and the range parameter. The slope of the phase $\phi(t)$ gives an indication of whether the energies in the impurity spectrum are predominantly positive or negative. In general, we observe that the amplitude near $t=0$ is characterized by an initial descent that is independent of scattering length and is only sensitive to $R^{*}$. The quantum evolution then displays oscillations on a time scale which is set by the Fermi time $\tau_{F}=1 / \varepsilon_{F}$. In $(\mathrm{c}, \mathrm{f})$, the dynamics is dominated by the attractive ground-state polaron, while for stronger attraction, the evolution can feature roughly equal contributions from the attractive and repulsive branches of the system, thus leading to pronounced oscillations in $|S(t)|$ and $\phi(t)$.

To quantity this further, we assume the attractive and repulsive branches are well-defined polaron quasiparticles, and consider the regime where both of their residues $-Z_{\text {att }}$ and $Z_{\text {rep }}$, respectively - are close to $1 / 2$. We can then analyse the Ramsey response in terms of the interference between the two polarons. Assuming that we can ignore the contribution from the continuum of states, we approximate the Ramsey response by

$$
S(t) \simeq Z_{\text {att }} e^{-i E_{\text {att }} t}+Z_{\text {rep }} e^{-i E_{\text {rep }} t},
$$

with $E_{\text {att }}\left(E_{\text {rep }}\right)$ the attractive (repulsive) polaron energy. As illustrated in Fig. 3, this approximation describes the response - in particular, the period of the beats - very well. Thus, the effect of $1 / k_{F} a$ and $k_{F} R^{*}$ on the dynamics may be simply estimated from their effect on the quasiparticle energies and residues. Sharp jumps in the phase accompany the regions where the amplitude approaches zero, and the direction of these jumps is the only feature of the dynamics that sensitively depends on the quasiparticle lifetime. Otherwise, we may assume both quasiparticles to be infinitely long lived. The validity of the approximation (21) hinges on the small residue of the states in the continuum that lies between the attractive and repulsive peaks. This feature is also observed in recent diagrammatic Monte Carlo calculations [34]. However, note that this is not necessarily true for arbitrary impurity mass, and indeed we find a larger weight in the 
continuum for a heavy impurity [15].

The behavior of the Ramsey response at times greatly exceeding $\tau_{F}$ is determined by the ground state of the impurity problem. If the attractive polaron is the ground state, this implies that there is a well-defined quasiparticle peak of zero width in the impurity spectral function. Hence, while all contributions to $S(t)$ in Eq. (20) originating from the higher-lying continuum of states interfere destructively and thus dephase, this single term becomes dominant. Therefore, in this limit, $|S(t)| \rightarrow Z_{\text {att }}$ and $\phi(t) \rightarrow E_{\text {att }} t$, corresponding to the formation of the attractive polaron. Since wave functions of the form (16) provide a good approximation to the residue and energy of the attractive polaron [27], we therefore expect that the TBM will accurately describe the long-time behavior of the Ramsey response for sufficiently weak interaction strengths where the attractive polaron is the ground state.
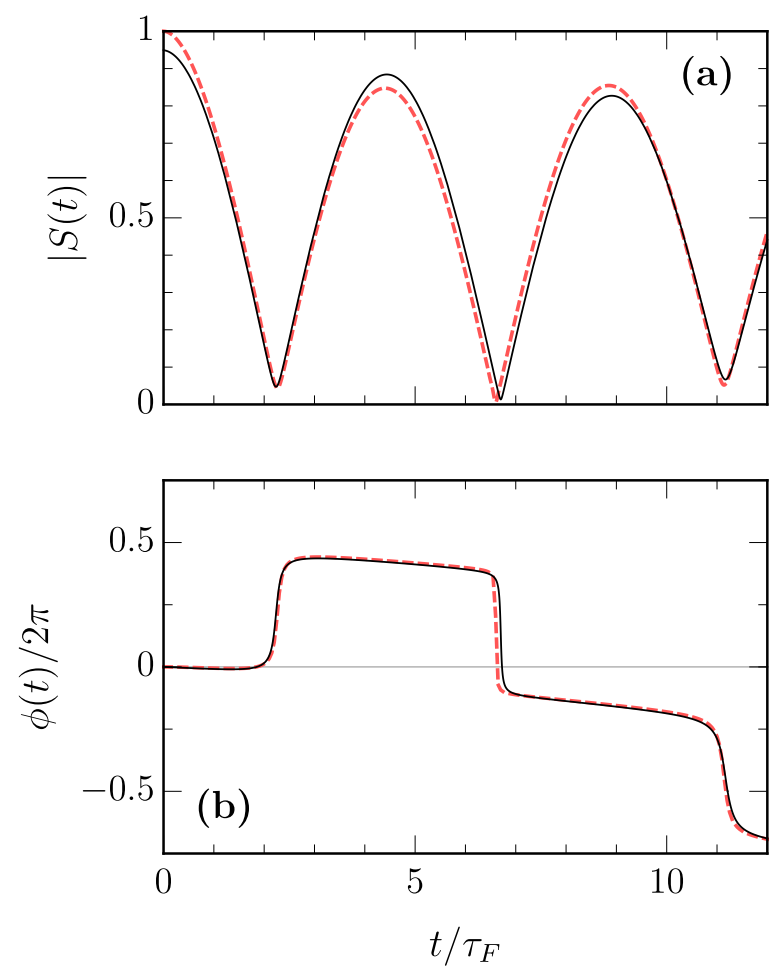

FIG. 3. (a) Amplitude and (b) phase of the Ramsey signal (dashed lines) together with the corresponding results from the approximation Eq. (21) (thin solid lines) for $1 / k_{F} a=$ 0.48 and $k_{F} R^{*}=1$. For these interaction parameters, we have $Z_{\text {att }} \approx 0.55$ and $Z_{\text {rep }} \approx 0.41$ [35]. We have added a small imaginary part $i \varepsilon_{F} / 33$ to the repulsive polaron energy to model the finite quasiparticle lifetime.

\section{B. Spectral function}

We now discuss how the dynamical response of the impurity to an interaction quench is related to the spectral response obtained using inverse rf spectroscopy. In the latter case, we start with impurities in the noninteracting spin $\downarrow$ state, and then apply an rf pulse that couples the two impurity spin states, as described by the Rabi term in the Hamiltonian - see Eq. (8). Assuming a weak pulse, $\Omega_{0} \ll \varepsilon_{F}$, such that it can be treated within linear response theory, the fraction of atoms transferred is directly proportional to the impurity spectral function. The protocol is illustrated in Fig. 1(b). The impurity spectral function has been measured in several ultracold atom experiments $[8,11,12]$. Theoretically, it has previously been treated within the renormalization group [36], in diagrammatic Monte Carlo [34], and within a $\mathrm{T}$ matrix approach [37]. The latter approach includes two-body correlations in the impurity wave function systematically, and is equivalent to the TBM calculation of the spectral function with one particle-hole excitation. However, the TBM is easier to extend to other types of impurity dynamics and to higher order correlations, as we show in Secs. V-VII.

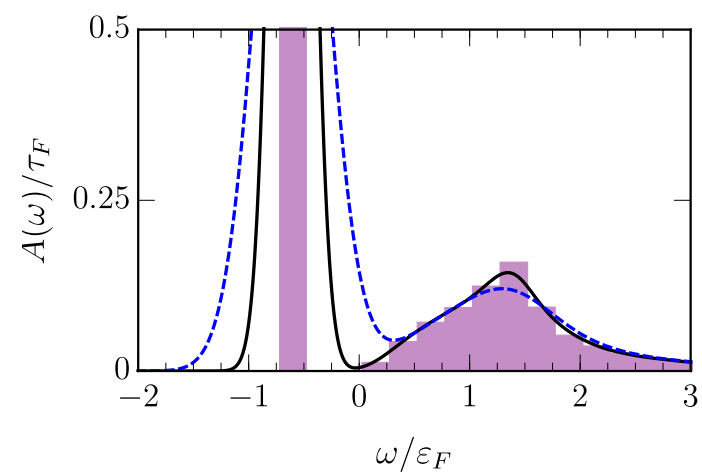

FIG. 4. Illustration of the decomposition of the spectral function at $1 / k_{F} a=k_{F} R^{*}=0$. The solid line is the convolved spectral function $I(\omega)$ according to Eq. (24) with a Gaussian width of $\sigma=0.15 E_{F}$ (solid) and $\sigma=0.3 E_{F}$ (dashed). The bars show $A(\omega)$ calculated according to Eq. (23), where the eigenvalues have been binned and the height of each bin set to $\sum_{j \in \text { bin }}\left|\left\langle\psi_{0} \mid \phi_{j}\right\rangle\right|^{2}$.

For a perfect quench in the dynamical problem, the Ramsey response (18) corresponds to the overlap between the time-evolved interacting and non-interacting states of the system. The spectral function $A(\omega)$ is then obtained from the Fourier transform of $S(t)$ [38]:

$$
A(\omega)=\operatorname{Re} \int_{0}^{\infty} \frac{d t}{\pi} e^{i \omega t} S(t) .
$$

This clearly illustrates the close connection between the energy spectrum and the dynamical response of the system to a quench of the system parameters.

Using Eq. (20), we can find an approximate spectral function within the TBM,

$$
A(\omega) \simeq \sum_{j}\left|\left\langle\psi_{0} \mid \phi_{j}\right\rangle\right|^{2} \underbrace{\int_{-\infty}^{\infty} \frac{d t}{2 \pi} e^{i \omega t} e^{-i\left(E_{j}-E_{0}\right) t}}_{\delta\left(\omega-E_{j}+E_{0}\right)} .
$$



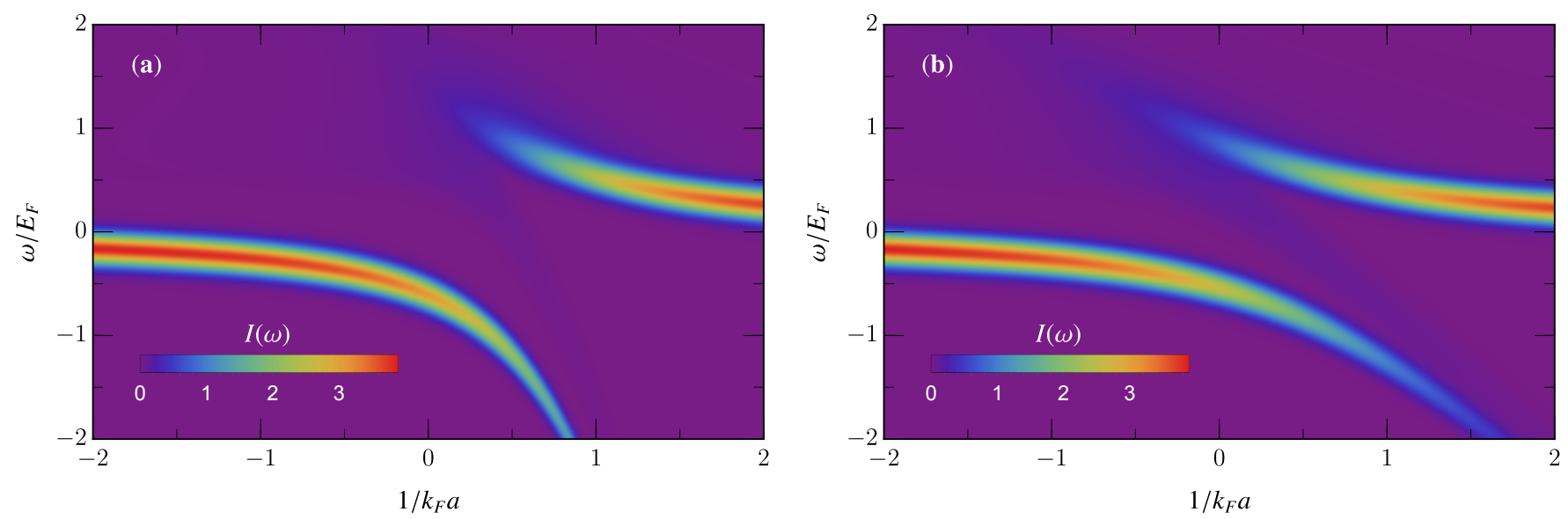

FIG. 5. Spectral function $I(\omega)$ calculated within the TBM with one particle-hole excitation for (a) $k_{F} R^{*}=0$ and (b) $k_{F} R^{*}=1$. The Gaussian width is taken to be $\sigma=0.1 E_{F}$. The dressed dimer state is the ground state rather than the attractive polaron when $1 / k_{F} a \gtrsim 0.9$ in (a) and $1 / k_{F} a \gtrsim 0.4$ in (b).

The Dirac delta function is easier to handle in the discretized basis if we first convolve $A(\omega)$ with a Gaussian of width $\sigma$ :

$$
\begin{aligned}
I(\omega) & =\int_{-\infty}^{\infty} d \omega^{\prime} A\left(\omega-\omega^{\prime}\right) g\left(\omega^{\prime}\right) \\
& =\sum_{j}\left|\left\langle\psi_{0} \mid \phi_{j}\right\rangle\right|^{2} g\left(\omega-E_{j}+E_{0}\right),
\end{aligned}
$$

where

$$
g(\omega)=\frac{1}{\sqrt{2 \pi} \sigma} e^{-\omega^{2} / 2 \sigma^{2}}
$$

Indeed, such a convolution mirrors experiment, where the spectral response is determined using rf pulses of a finite duration and hence a non-zero width in frequency space. This width can typically be well approximated by a Gaussian.

In Fig. 4, we illustrate the idea behind the method: First we evaluate the raw spectrum of energy eigenvalues and corresponding residues, which yields a large number of discrete peaks of variable heights. The convolved spectral function, $I(\omega)$, on the other hand, is a smooth function of frequency and is what would be observed in experiment. Such a spectral convolution is easier to generate using the TBM compared to the standard T matrix approach [37].

We show the results of this procedure in Fig. 5 for two values of $k_{F} R^{*}$. We see that the spectrum in both cases is dominated by the attractive and repulsive polaron quasiparticles at positive and negative energy, respectively. In between, there is a broad continuum of states which all have a very small wave function overlap with the non-interacting impurity state. In particular, once $1 / k_{F} a \gtrsim 1$, the spectral weight of the continuum is essentially negligible, as was also observed in Ref. [34].

The main effect of the range parameter $R^{*}$ is to shift the energies of the polaron branches closer to zero, especially in the unitary regime, and to increase the lifetime of the repulsive polaron such that it can be well-defined even on the attractive side of the resonance [39]. It also affects the character of the impurity ground state: With increasing $1 / k_{F} a$, the impurity eventually undergoes a sharp transition from an attractive polaron to a dressed dimer [27-29], and this transition occurs at lower $1 / k_{F} a$ for larger $k_{F} R^{*}$. However, this is not captured by the TBM with one particle-hole excitation, since the attractive polaron always remains the ground state at this level of truncation.

\section{Short-time dynamics}

We now turn to the limiting behaviour of $S(t)$ at short times when $t \lesssim \tau_{F}$. Away from resonance, the results presented in the following furthermore require $t \ll 2 m_{r} a^{2}$ when $\left|k_{F} a\right| \ll 1$. We start by formally Taylor expanding the time evolution operator in Eq. (18), which yields:

$$
S(t) \simeq 1-i\left\langle\psi_{0}|\delta \hat{H}| \psi_{0}\right\rangle t-\frac{1}{2}\left\langle\psi_{0}\left|(\delta \hat{H})^{2}\right| \psi_{0}\right\rangle t^{2},
$$

where $\delta \hat{H}=\hat{H}_{\text {int }}-E_{0}$. Using Eq. (22), we see that the first term yields the usual sum rule for the spectral function:

$$
\int_{-\infty}^{\infty} d \omega A(\omega)=1
$$

For the second term, using the two-channel Hamiltonian (4), we simply obtain:

$$
\left\langle\psi_{0}|\delta \hat{H}| \psi_{0}\right\rangle=g\left\langle\mathrm{FS}\left|\hat{c}_{\mathbf{0} \uparrow} \sum_{\mathbf{q}} \hat{d}_{\mathbf{q}}^{\dagger} \hat{f}_{\mathbf{q}}\right| \mathrm{FS}\right\rangle=0 .
$$

Thus, the leading order behavior of $S(t)$ is determined by the last term

$$
\left\langle\psi_{0}\left|(\delta \hat{H})^{2}\right| \psi_{0}\right\rangle=g^{2} \sum_{|\mathbf{q}|<k_{F}}=\frac{g^{2} k_{F}^{3}}{6 \pi^{2}},
$$


which finally gives:

$$
S(t) \simeq 1-\frac{k_{F}^{3} t^{2}}{12 \pi R^{*} m_{r}^{2}}
$$

These results yield an additional set of sum rules for the spectral function of the impurity (see also Ref. [40]):

$$
\begin{aligned}
\int_{-\infty}^{\infty} d \omega \omega A(\omega) & =0 \\
\int_{-\infty}^{\infty} d \omega \omega^{2} A(\omega) & =\frac{k_{F}^{3}}{6 \pi R^{*} m_{r}^{2}} .
\end{aligned}
$$

Note that in the limit of a broad resonance where $g \rightarrow \infty$, Eq. (32) diverges and there is no well-defined short-time parabolic decay of $|S(t)|$. As such, it does not resemble the initial decay of the Loschmidt echo expected for quantum systems in this case. Indeed, even when $g$ is finite, we find that terms involving higher powers of $\delta \hat{H}$ are divergent, e.g., for the next order term, we obtain

$$
\left\langle\psi_{0}\left|(\delta \hat{H})^{3}\right| \psi_{0}\right\rangle=g^{2} \sum_{|\mathbf{q}|<k_{F}}\left(\epsilon_{\mathbf{q}, M}-\epsilon_{\mathbf{q}, L i}+\nu\right),
$$

which clearly diverges for short-range interactions since $\nu \sim \Lambda \rightarrow \infty$.

The origin of these divergences is the non-analytic behavior of the many-body wave function when the distance between the impurity and a majority fermion goes to zero. One thus needs to isolate the high-frequency behavior of $A(\omega)$ in order to address the short-time dynamics in the presence of short-range interactions.

From the Green's function $G(\omega)$ for an impurity at zero momentum, we have $A(\omega)=-\operatorname{Im}[G(\omega)] / \pi$, where we can, in turn, write the Green's function in terms of the self energy $\Sigma(\omega)$ :

$$
G(\omega)=[\omega-\Sigma(\omega)]^{-1}
$$

In the limit $\omega \rightarrow \infty$, we can neglect non-trivial effects of the Fermi medium, i.e., the self-energy is dominated by two-body scattering, giving:

$$
\Sigma(\omega) \simeq n \mathcal{T}(\omega)
$$

where the medium density $n=\frac{k_{F}^{3}}{6 \pi^{2}}$ and the two-body T matrix for $\omega>0$ is

$$
\mathcal{T}(\omega)=\frac{2 \pi}{m_{r}}\left(a^{-1}+2 m_{r} \omega R^{*}+i \sqrt{2 m_{r} \omega}\right)^{-1} .
$$

Thus, the high-frequency limit of the spectral function is contained in the expression

$$
A(\omega) \simeq \frac{k_{F}^{3}}{3 \pi^{2} \sqrt{2 m_{r}^{3}} \omega^{5 / 2}} \frac{1}{1+2 R^{*} / a+2 m_{r} \omega R^{* 2}} .
$$

Focussing first on the case $R^{*}=0$, the leading order correction to $S(t)$ in the limit $t \rightarrow 0$ can be determined from the integral:

$$
\int_{\tilde{\omega}}^{\infty} d \omega A(\omega)\left(e^{-i \omega t}-1+i \omega t\right)
$$

where $\tilde{\omega}$ is a large frequency scale that can be sent to infinity at the end of the calculation. Inserting Eq. (37), we finally obtain

$$
\begin{aligned}
S(t) & \simeq 1+\frac{k_{F}^{3} t^{3 / 2}}{3 \pi^{2} \sqrt{2 m_{r}^{3}}} \lim _{t \rightarrow 0}\left[\int_{\tilde{\omega} t}^{\infty} d \omega \frac{\left(e^{-i \omega}-1+i \omega\right)}{\omega^{5 / 2}}\right] \\
& =1-\frac{2 \sqrt{\pi}(1-i) k_{F}^{3}}{9 \pi^{2} \sqrt{m_{r}^{3}}} t^{3 / 2}
\end{aligned}
$$

for $t>0$, with $S(-t)=S^{*}(t)$. We have thus succeeded in deriving Eq. (2) from the introduction. This universal, non-analytic expression for the short-time behavior is a key result of this paper.

For the case where $R^{*}>0$, the non-analytic behavior appears in the next order term of $S(t)$, which can be obtained from the integral:

$$
\begin{aligned}
& \lim _{t \rightarrow 0} \int_{\tilde{\omega}}^{\infty} d \omega A(\omega)\left(e^{-i \omega t}-1+i \omega t+\frac{1}{2} \omega^{2} t^{2}\right) \\
& =\frac{k_{F}^{3} t^{5 / 2}}{6 \pi^{2} R^{*} \sqrt{2 m_{r}^{5}}} \lim _{t \rightarrow 0}\left[\int_{\tilde{\omega} t}^{\infty} d \omega \frac{\left(e^{-i \omega}-1+i \omega+\frac{1}{2} \omega^{2}\right)}{\omega^{7 / 2}}\right] .
\end{aligned}
$$

Evaluating the integral, we thus obtain for the short-time expansion of $S(t)$ :

$$
S(t) \simeq 1-\frac{k_{F}^{3} t^{2}}{12 \pi R^{*} m_{r}^{2}}+\frac{2 \sqrt{\pi}(1+i) k_{F}^{3}}{45 \pi^{2} R^{* 2} \sqrt{m_{r}^{5}}} t^{5 / 2},
$$

which demonstrates Eq. (3) from the introduction. At first glance, one might expect the short-time behavior of the Ramsey response (or high-frequency tail of the spectral function) to be connected to the Tan contact [41]. However, we emphasize that the Tan contact governs the large-frequency behavior of the occupied spectral function in the equilibrium system, not the full spectral function probed here.

In the weak coupling limit $\left|k_{F} a\right| \ll 1$, the form of the $\mathrm{T}$ matrix allows us to compute the leading corrections to Eqs. $(39,40)$, which are respectively given by

$$
-\frac{2 \sqrt{\pi}(1+i) k_{F}^{3}}{45 \pi^{2} a^{2} \sqrt{m_{r}^{5}}} t^{5 / 2}, \quad-\frac{4 \sqrt{\pi}(1-i) k_{F}^{3}}{315 \pi^{2} R^{*} 3 a \sqrt{m_{r}^{7}}} t^{7 / 2} .
$$

These corrections yield the lowest orders at which the scattering length enters the Ramsey response.

Our results (39) and (40) are valid also for a finite temperature $T$ provided that the time $t$ is shorter than the characteristic time scale $1 / T$ at which thermal effects become relevant. Likewise, for a finite impurity momentum $\mathbf{p}$, it is clear from the form of the $\mathrm{T}$ matrix that there always exists a frequency above which $\epsilon_{\mathbf{p} \text {,im }}$ is negligible and therefore our results remain unchanged for $t \ll 1 / \epsilon_{\mathbf{p}, \mathrm{im}}$. On the other hand our results are, in general, sensitive to the preparation of the initial state.

\section{RABI OSCILLATIONS}

Another important example of coherent impurity dynamics is the Rabi oscillations between $\downarrow$ and $\uparrow$ impurity 

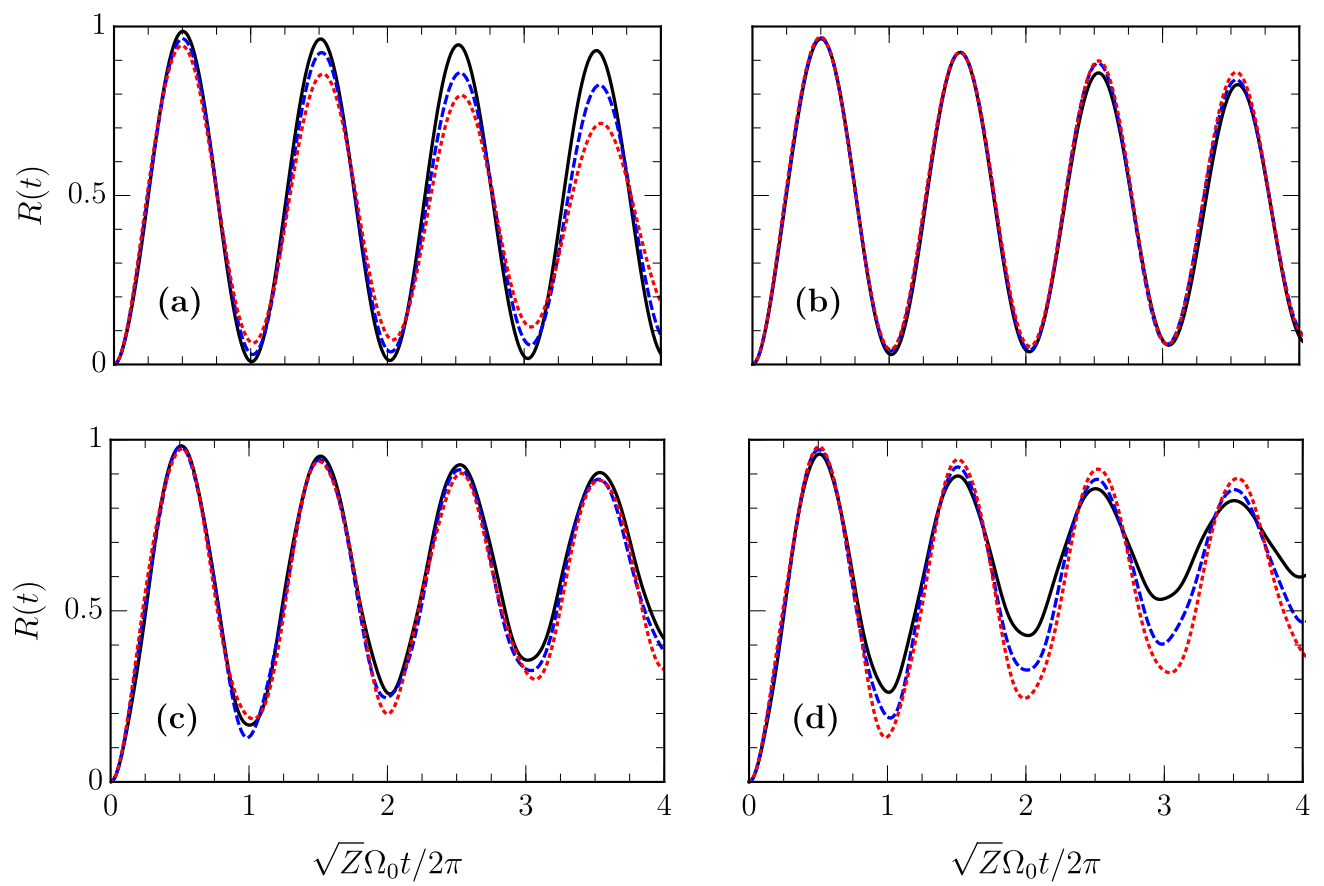

FIG. 6. Occupation of the $\uparrow$ impurity state, $R(t)$, as a function of time under a rf driving field of strength $\Omega_{0}$ that couples to the attractive $\uparrow$ polaron (a,b) and the repulsive $\uparrow$ polaron (c,d). (a) The response at unitarity and $R^{*}=0$ for different Rabi frequencies $\Omega_{0}=0.5 \varepsilon_{F}$ (black solid), $0.75 \varepsilon_{F}$ (blue dashed), and $\varepsilon_{F}$ (red dotted). (b) The response at fixed $\Omega_{0}=0.75 \varepsilon_{F}$ for different $k_{F} R^{*}=0,0.5,1$ with $1 / k_{F} a=0,-0.277,-0.505$, (black solid, blue dashed, and red dotted), respectively. (c) Same Rabi frequencies as in (a), but with $k_{F} R^{*}=1$ and $1 / k_{F} a=1$. (d) The response at fixed $\Omega_{0}=0.75 \varepsilon_{F}$ and $1 / k_{F} a=1$ for different $k_{F} R^{*}=0.1,0.5,1$, (black solid, blue dashed, and red dotted), respectively. The conditions are arranged such that the residue of the $\uparrow$ polaron is always $Z \simeq 0.784$.

states that are driven by a continuous rf field. The presence of the Fermi medium has an observable effect on the oscillations when the spin- $\uparrow$ state is strongly interacting with the majority fermions. For concreteness, we assume that the impurity atom is initially in the noninteracting $\downarrow$ ground state, i.e., $\left|\psi_{R}(t=0)\right\rangle=\hat{c}_{0 \downarrow}|\mathrm{FS}\rangle$. Then, at times $t \geq 0$ the spin- $\downarrow$ impurity is coupled to the interacting state by the Rabi term in the Hamiltonian - see Eq. (8). By adjusting the rf detuning $\delta$ to match the attractive or repulsive polaron energy, we can address either of these quasiparticle branches. Unlike in the perfect quench Ramsey response, Rabi oscillations require us to take both impurity spin states explicitly into account, and we thus employ the TBM with wave functions of the form (16) to describe the dynamics. Specifically, we are interested in the spin- $\uparrow$ population $N_{\uparrow}=\left\langle\psi_{R}(t)\left|\sum_{\mathbf{k}} \hat{c}_{\mathbf{k} \uparrow}^{\dagger} \hat{c}_{\mathbf{k} \uparrow}\right| \psi_{R}(t)\right\rangle$, where the timedependent wave function $\left|\psi_{R}(t)\right\rangle=e^{-i \hat{H} t} \hat{c}_{\mathbf{0}}|\mathrm{FS}\rangle$.

In Fig. 6, we show the relative occupation of the spin$\uparrow$ impurity state, $R(t)=N_{\uparrow} /\left(N_{\uparrow}+N_{\downarrow}\right)$, as a function of time for Rabi frequencies typical in experiment. In panels $(\mathrm{a}, \mathrm{b})$, the rf field addresses the attractive polaron, while $(\mathrm{c}, \mathrm{d})$ show the results for the repulsive polaron. In all cases, regardless of the interactions or the range parameter, we observe that the spin- $\uparrow$ occupation displays a damped oscillatory behavior with period $2 \pi /\left(\sqrt{Z} \Omega_{0}\right)$.
In other words, the angular frequency of the Rabi oscillation in the presence of the Fermi medium is reduced by a factor $\sqrt{Z}$ compared with that expected for a noninteracting spin- $\uparrow$ state. This observation is consistent with the spin- $\uparrow$ spectral function being dominated by the quasiparticle peaks, as in Fig. 5. Specifically, if we assume that the spectrum only contains the addressed quasiparticle, then one obtains [11]

$$
R(t) \simeq \sin ^{2}\left(\sqrt{Z} \Omega_{0} t / 2\right) .
$$

This reduction of the Rabi frequency has been used as a means to experimentally access the polaron residue [11].

The manner of damping and the functional form of the Rabi oscillations appears different in the four panels of Fig. 6. In $(\mathrm{a}, \mathrm{c})$ we investigate the effect of changing the Rabi frequency $\Omega_{0}$ at fixed interaction strength, whereas in $(b, d)$ we change the interaction strength while keeping $\Omega_{0}$ fixed. We see that when we address the attractive polaron, the damping is sensitive to the bare Rabi frequency, but quite insensitive to the precise interaction parameters. The opposite appears to be the case for the repulsive polaron, where the finite quasiparticle lifetime dominates the damping and depends sensitively on the interaction parameters $1 / k_{F} a$ and $k_{F} R^{*}$. This prediction is, in principle, straightforward to test experimentally for sufficiently low temperatures. In practice, there will also be damping due to thermal effects once $t>1 / T$. 

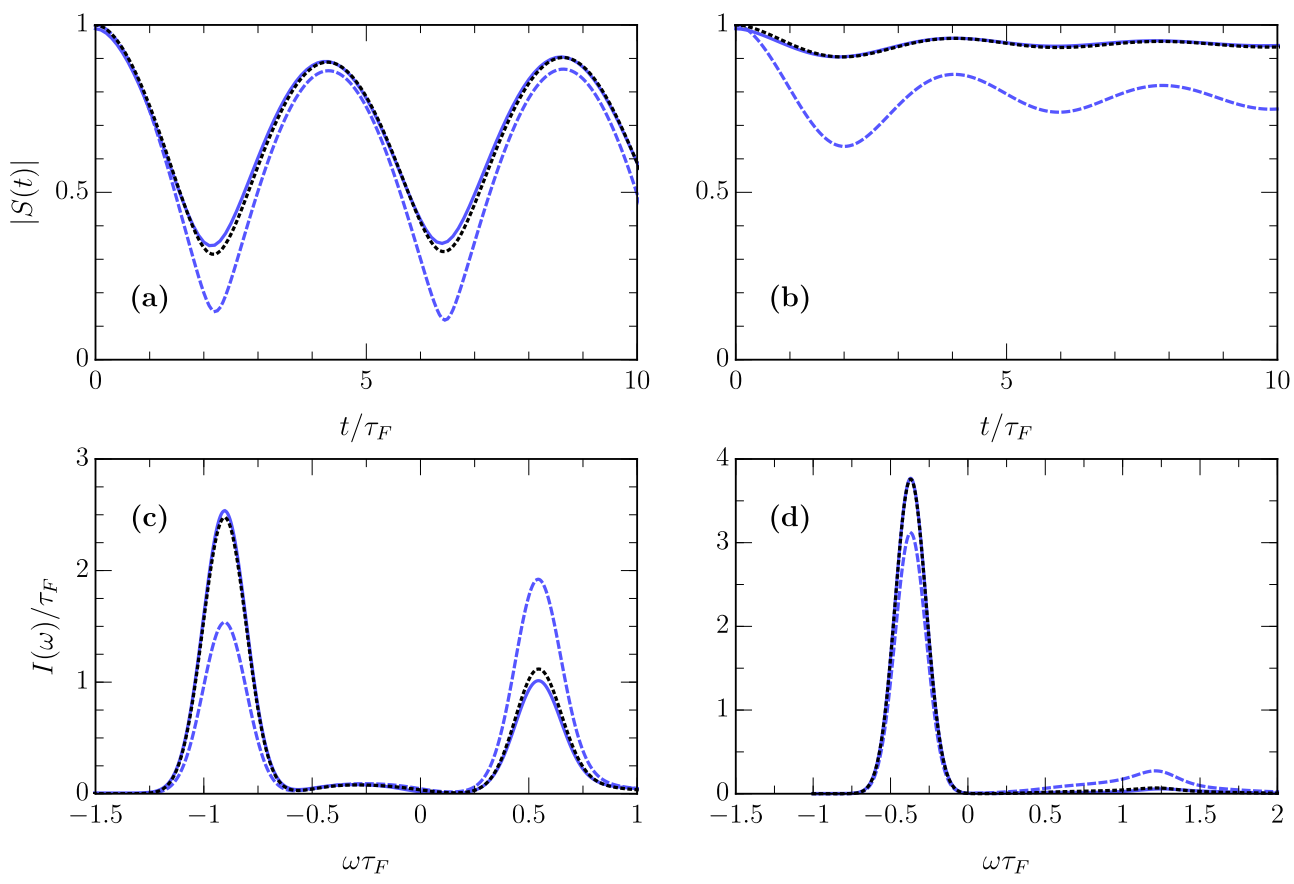

FIG. 7. The Ramsey response $S(t)$ and corresponding convolved Fourier transform $I(\omega)$, with frequency broadening $\sigma=0.1 \varepsilon_{F}$. The interactions in the strongly interacting regime are (a,c) repulsive with $1 / k_{F} a=0.6$, and (b,d) attractive with $1 / k_{F} a=-0.5$. The range parameter is always taken to be $k_{F} R^{*}=1$. We show the result of a perfect quench to strong interactions (dashed), a state preparation with $t_{\mathrm{rf}}=3 \tau_{F}$ at $1 / k_{F} a_{0}=-2$ (solid), and the approximation Eq. (42) (dotted). In the second case, we include a wait time of $0.5 \tau_{F}$ at $1 / k_{F} a_{0}$ just after (before) the first (second) rf pulse to mimic the quantum state preparation in experiment.

\section{QUANTUM STATE PREPARATION}

For the Ramsey response $S(t)$, we have thus far considered the situation of a perfect quench, where there is no effect of the medium during the $\pi / 2 \mathrm{rf}$ pulses, either because the pulses are infinitely fast or because the impurity-medium interactions are switched off during the pulses. However, it is important to understand how such 'residual' interactions with the medium affect the impurity dynamics since a perfect quench is challenging to achieve in practice [15]. Furthermore, one could in principle use the residual interactions to tailor the initial state and engineer the desired dynamical response.

To assess this effect in detail, we once again calculate the response following the rf sequence in Fig. 1, but this time we consider the full Hamiltonian and wave function (16) rather than the decoupled spin- $\uparrow$ versions. We address the attractive polaron branch for interaction $1 / k_{F} a_{0}$ during the rf pulses by setting the rf detuning $\delta=E_{\text {att }}$, and then we extract $S(t)$ as defined in Eq. (17). We use the Rabi frequency $\Omega_{0} / \varepsilon_{F}=\pi / 6$, which is typical in experiment. In a previous work [15], we employed such an approach for the case of a heavy impurity and weak interactions, $\left|k_{F} a_{0}\right| \ll 1$, during the rf pulses. Here, we consider stronger interactions on the attractive side of resonance for the case of equal masses, as shown in Fig. 7. Compared to the result for the perfect quench, we see that the residual attractive interactions clearly favour the attractive polaron branch in the spectral function. This results in a decrease in the amplitude of oscillations in the time domain and an overall increase in the contrast $|S(t)|$ for the interactions considered. In principle, one could have a scenario where the residual interactions increase the amplitude of oscillations, but this requires a larger $1 / k_{F} a$, where the TBM with a single particlehole excitation becomes increasingly inaccurate for the attractive branch.

Further insight can be gained by restricting ourselves to the spin- $\uparrow$ subspace and considering the approximate expression

$$
S(t) \simeq e^{i E_{0} t}\left\langle\psi_{\text {att }}\left|e^{-i \hat{H}_{\text {int }} t}\right| \psi_{\text {att }}\right\rangle,
$$

where $\left|\psi_{\text {att }}\right\rangle$ is the attractive polaron state at interaction parameter $1 / k_{F} a_{0}$ during the rf pulse. Referring to Fig. 7, we see that this approximation well reproduces both the dynamical and spectral response obtained from the full calculation. Thus, we conclude that the main effect of the interactions during the rf pulses is to adiabatically prepare attractive polaron states starting from the non-interacting wave function. We expect this situation to hold provided the pulse duration is longer than the Fermi time $\tau_{F}$. In the limit $\Omega \rightarrow \infty$, where the $\pi / 2 \mathrm{rf}$ pulses become infinitely fast, we should recover the perfect quench scenario from Sec. IV. In general, one could consider preparing other initial, or reference, wave functions, which would shape the final Ramsey response. 

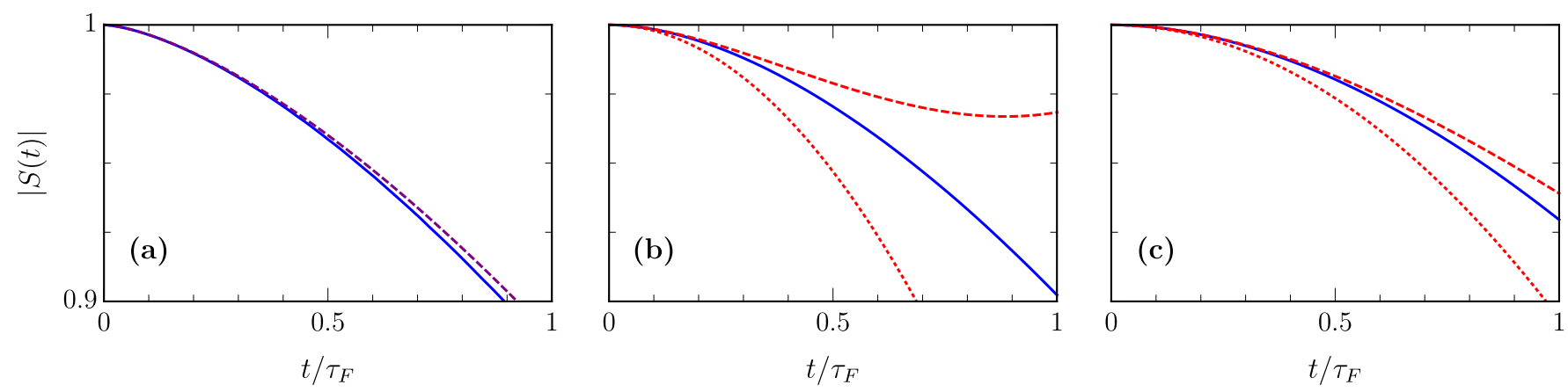

FIG. 8. Short time behavior of the Ramsey contrast $|S(t)|$ at unitarity for different values of the range parameter: (a) $k_{F} R^{*}=0$, (b) $k_{F} R^{*}=1 / 2$, and (c) $k_{F} R^{*}=1$. The result of the TBM where the wave functions are restricted to one particle-hole excitation as in Eq. (16) is shown as a solid line. In (a) this is compared with the short-time expansion for a broad resonance, Eq. (39) (dashed line). In (b,c) the short-time expansion at finite $R^{*}$ restricted to the $\mathcal{O}\left(t^{2}\right)$ correction, Eq. (30), is shown as a dotted line, and the short-time expansion including the $\mathcal{O}\left(t^{5 / 2}\right)$ correction, Eq. (40), as a dashed line. On this scale, the result of the TBM with two particle-hole excitations is indistinguishable from the solid line.
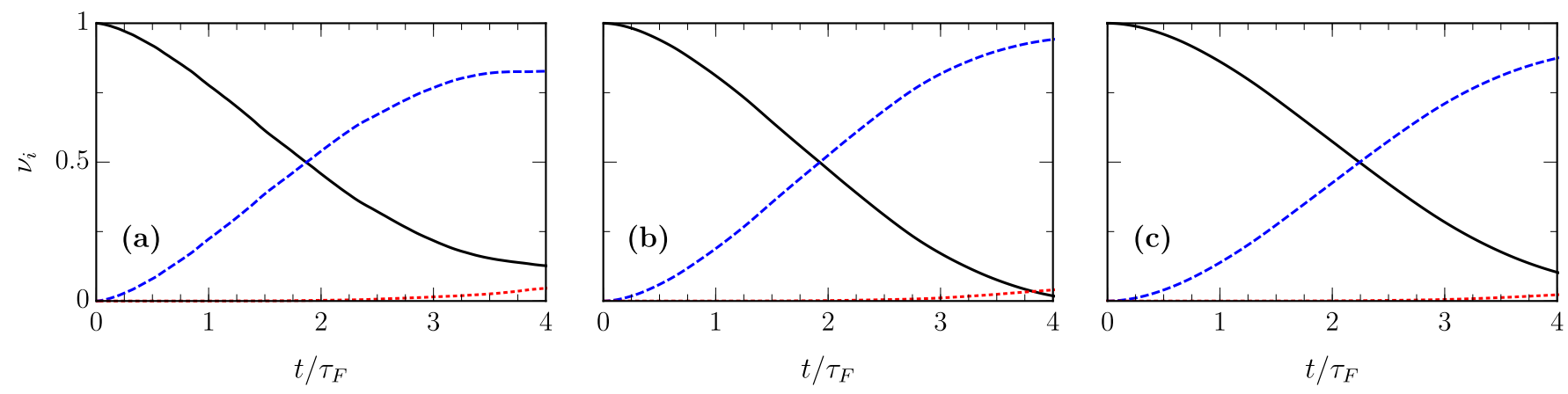

FIG. 9. Probability of $\psi(t)$ to be in states with 0 (solid), 1 (dashed), and 2 (dotted) holes excited from the Fermi sea. The results are shown at unitarity for (a) $k_{F} R^{*}=0$, (b) $k_{F} R^{*}=1 / 2$, and (c) $k_{F} R^{*}=1$.

\section{MULTIPLE PARTICLE-HOLE EXCITATIONS}

We now discuss the role played by multiple particlehole excitations in the impurity dynamics, quantified here by the Ramsey contrast for the perfect quench. Going beyond the single particle-hole approximation in the TBM is in general a complicated problem, as the size of the truncated subspace grows exponentially with the number of excitations of the Fermi sea. Thus, in this section we focus on a simpler problem than what has been described so far, namely that of a static (infinitely heavy) impurity particle. In this case, the angular degrees of freedom can be integrated out, allowing us to extend the wave function (16) to two particle-hole excitations (see Appendix A for the mathematical details).

The static impurity problem may be solved exactly since it reduces to the problem of a single particle in the presence of a fixed potential. At the same time, we expect the static impurity to constitute a worst case scenario for the TBM since it features the orthogonality catastrophe [14], where there is no well-defined quasiparticle (i.e., the residue $Z=0$ ) and one has an infinite number of low-energy excitations. We previously compared the exact solution with the TBM for one particle-hole excitation and found excellent agreement for short times up to order $10 \tau_{F}$ near unitarity [15]. Here, we analyse the structure of the wave function and estimate the timescale at which multiple particle-hole excitations appear for different range parameters $k_{F} R^{*}$.

Consider first the Ramsey response at times $t \lesssim \tau_{F}$. In this case, the analytic expressions for $|S(t)|$ at short times - Eq. (39) for $R^{*}=0$ and Eq. (40) for $R^{*}>0$ - were derived from the observation that the short-time dynamics is governed by large frequencies and thus twobody physics. Since the wave function with one particlehole excitation, Eq. (16), explicitly includes the processes constituting the two-body scattering $T$ matrix, we expect the short-time dynamics to be well captured by this wave function. In Fig. 8 we show the Ramsey contrast at unitarity for $k_{F} R^{*}=0,1 / 2$, and 1 , and indeed we observe that both the initial $t^{3 / 2}$ decrease of the contrast for $k_{F} R^{*}=0$, and the $t^{2}$ decrease plus $t^{5 / 2}$ correction for finite $k_{F} R^{*}$ are well captured by the TBM. At times up to $\tau_{F}$, we furthermore find perfect agreement between the results of diagonalizing the wave functions with one and two particle-hole excitations, as the results are iden- 
tical within our numerical error (which, for $t \lesssim 4 \tau_{F}$, we estimate to be less than $0.01 \%$ in the one particle-hole TBM and less than $1 \%$ for $|S(t)|$ in the two particle-hole TBM).

At times exceeding $\tau_{F}$, eventually multiple particlehole excitations become important. In order to quantify the contribution from the different terms in the variational wave function, we project $\psi(t)$ including two particle-hole excitations (App. A) onto basis states with a fixed number - 0,1 , or 2 - of holes excited from the Fermi sea:

$$
\begin{aligned}
\nu_{0} & =\left|\left\langle\mathrm{FS}\left|\hat{c}_{\mathbf{0} \uparrow}\right| \psi(t)\right\rangle\right|^{2}, \\
\nu_{1} & =\sum_{\mathbf{q}}\left|\left\langle\mathrm{FS}\left|\hat{f}_{\mathbf{q}}^{\dagger} \hat{d}_{\mathbf{q}}\right| \psi(t)\right\rangle\right|^{2}+\sum_{\mathbf{k}, \mathbf{q}}\left|\left\langle\mathrm{FS}\left|\hat{f}_{\mathbf{q}}^{\dagger} \hat{f}_{\mathbf{k}} \hat{c}_{\mathbf{q}-\mathbf{k} \uparrow}\right| \psi(t)\right\rangle\right|^{2}, \\
\nu_{2} & =\frac{1}{2} \sum_{\mathbf{k}, \mathbf{q}_{1}, \mathbf{q}_{2}}\left|\left\langle\mathrm{FS}\left|\hat{f}_{\mathbf{q}_{1}}^{\dagger} \hat{f}_{\mathbf{q}_{2}}^{\dagger} \hat{f}_{\mathbf{k}} \hat{d}_{\mathbf{q}_{1}+\mathbf{q}_{2}-\mathbf{k}}\right| \psi(t)\right\rangle\right|^{2} \\
& +\frac{1}{4} \sum_{\mathbf{k}_{1}, \mathbf{k}_{2}, \mathbf{q}_{1}, \mathbf{q}_{2}}\left|\left\langle\mathrm{FS}\left|\hat{f}_{\mathbf{q}_{1}}^{\dagger} \hat{f}_{\mathbf{q}_{2}}^{\dagger} \hat{f}_{\mathbf{k}_{1}} \hat{f}_{\mathbf{k}_{2}} \hat{c}_{\mathbf{q}_{1}+\mathbf{q}_{2}-\mathbf{k}_{1}-\mathbf{k}_{2} \uparrow}\right| \psi(t)\right\rangle\right|^{2} .
\end{aligned}
$$

As previously, $\mathbf{k}, \mathbf{k}_{1}, \mathbf{k}_{2}$ denote particles above the Fermi sea and $\mathbf{q}, \mathbf{q}_{1}, \mathbf{q}_{2}$ holes. Note that we have $\nu_{0}=|S(t)|^{2}$ and $\nu_{0}+\nu_{1}+\nu_{2}=1$. In Fig. 9 we display these quantities at unitarity for various values of the resonance range. We clearly see that two particle-hole excitations remain insignificant even at several times the Fermi time. Furthermore, this result is independent of the resonance range, and thus it is insensitive to the precise power-law behavior at short times.

Since the static impurity is a worst case scenario, we expect the TBM for wave functions (16) to accurately describe the short-time dynamics also for a mobile impurity.

\section{OUTLOOK}

In this work, we have shown how the truncated basis method can be used to determine the coherent quantum evolution of an impurity for a variety of scenarios, including the dynamical response to a suddenly introduced impurity, and the Rabi oscillations between a polaron quasiparticle and a non-interacting impurity state. We have furthermore explored the connection between impurity dynamics and the spectral function, as well as the possibility of preparing different quantum many-body states. We emphasize that the method is quite general, and allows one to investigate more complicated dynamical protocols. For instance, a spin-echo sequence can be efficiently modelled as a series of time-evolution operators, each of which are evaluated within the truncated basis.

A key result is the exact short-time Ramsey response, i.e., for $t \lesssim \tau_{F}$. Surprisingly, this was found to depend non-analytically on time, which is a direct consequence of the need to renormalize the short-range interactions. Additionally, the response to leading order does not depend on the scattering length, although the regime of validity of the short-time expansion does. We also note that our short-time expansion is not affected by temperature in a degenerate gas, since the time-scale associated with thermal excitations is longer than the Fermi time. Therefore, our predictions can be tested in current precision experiments on ultracold atomic gases out of equilibrium [15].

In the long-time limit $t \gg \tau_{F}$ (but still $t \lesssim 1 / T$ ), we have argued that if the ground state of the interacting impurity is a well-defined attractive polaron, the Ramsey response will be dominated by the corresponding single peak in the spectral function. In turn, this limit will be well captured by the TBM, as it describes both the energy and residue of the attractive polaron very well [27]. While the TBM thus captures both the short- and the longtime coherent impurity dynamics well, the evolution at intermediate times presents an outstanding challenge to theories of strongly correlated quantum matter.

For a static impurity, we have shown that multiple particle-hole excitations only become prominent in the Ramsey response at time scales significantly exceeding the Fermi time. Thus, while we expect a power-law decay of the contrast $|S(t)|$ due to the orthogonality catastrophe, this is driven by low-energy excitations which are only relevant at long times. In the opposite limit of a light impurity, the Ramsey response will likely develop exotic few-body correlations. In this case, for sufficiently large mass ratio, the system is predicted to feature universal trimers [42] with associated resonant few-body interactions [43], or even Efimov trimers [44] and tetramers [45]. The theoretical description of such systems would thus require one to go to a truncated Hilbert space featuring multiple excitations of the medium.

An open question is how to extend the present work to non-zero temperature, where one requires a thermal average over all initial states. In particular, it would be interesting to understand how the impurity dynamics evolves from the quantum short-time regime to the thermal long-time limit. When the interactions are weak $\left|k_{F} a\right|<1$, the long-time decoherence due to thermal fluctuations is well described using a Fermi liquid calculation for quasiparticle scattering [46]. A major simplification is to approximate the impurity as being effectively fixed by the thermal excitations of the medium, in which case the Ramsey response may be obtained exactly using a functional determinant approach [15]. However, such an approximation is only expected to be reasonable when $\varepsilon_{F} m / m_{\mathrm{im}}<T<\varepsilon_{F}$, i.e., for sufficiently heavy impurities and sufficiently high temperatures [47].

Finally, the framework developed here is not limited to a single-component fermionic medium. As an example, we have recently calculated the spectral response to an rf pulse for an impurity in a Bose-Einstein condensate: Here we obtained a very good agreement between the TBM, containing up to two Bogoliubov excitations of the condensate, and the experimental measurements [48]. Likewise, the TBM could be applied to the problem of an impurity in a two-component Fermi gas across the 
BCS-BEC crossover [49, 50]. A particularly interesting future application would be to the investigation of threebody correlations in a Bose-Einstein condensate that is suddenly quenched to unitarity, as in experiment [51].

\section{ACKNOWLEDGMENTS}

We gratefully acknowledge fruitful discussions with Masud Haque, Richard Schmidt, Georg Bruun, Marko Cetina, Jook Walraven, and Rudi Grimm.

\section{Appendix A: Variational wave functions with two particle-hole exciations}

In this appendix, we present the variational equations for a wave function with two particle-hole excitations:

$$
\begin{aligned}
|\psi\rangle= & {\left[\alpha_{0} \hat{c}_{\mathbf{0} \uparrow}^{\dagger}+\sum_{\mathbf{q}} \alpha_{\mathbf{q}} \hat{d}_{\mathbf{q}}^{\dagger} \hat{f}_{\mathbf{q}}+\sum_{\mathbf{k}, \mathbf{q}} \alpha_{\mathbf{k q}} \hat{c}_{\mathbf{q}-\mathbf{k} \uparrow}^{\dagger} \hat{f}_{\mathbf{k}}^{\dagger} \hat{f}_{\mathbf{q}}+\sum_{\mathbf{k}, \mathbf{q}_{1}, \mathbf{q}_{2}} \alpha_{\mathbf{k q}_{1} \mathbf{q}_{2}} \hat{d}_{\mathbf{q}_{1}+\mathbf{q}_{2}-\mathbf{k}}^{\dagger} \hat{f}_{\mathbf{k}}^{\dagger} \hat{f}_{\mathbf{q}_{1}} \hat{f}_{\mathbf{q}_{2}}\right.} \\
& \left.+\sum_{\mathbf{k}_{1}, \mathbf{k}_{2}, \mathbf{q}_{1}, \mathbf{q}_{2}} \alpha_{\mathbf{k}_{1} \mathbf{k}_{2} \mathbf{q}_{1} \mathbf{q}_{2}} \hat{c}_{\mathbf{q}_{1}+\mathbf{q}_{2}-\mathbf{k}_{1}-\mathbf{k}_{2} \uparrow}^{\dagger} \hat{f}_{\mathbf{k}_{1}}^{\dagger} \hat{f}_{\mathbf{k}_{2}}^{\dagger} \hat{f}_{\mathbf{q}_{1}} \hat{f}_{\mathbf{q}_{2}}\right]|\mathrm{FS}\rangle,
\end{aligned}
$$

which provides a natural extension of the wave function (16). The variational equations then become

$$
\begin{aligned}
\left(E-E_{0}\right) \alpha_{0} & =g \sum_{\mathbf{q}} \alpha_{\mathbf{q}} \\
\left(E-E_{0}\right) \alpha_{\mathbf{q}} & =\left(\epsilon_{\mathbf{q}, \mathrm{M}}-\epsilon_{\mathbf{q}}+\nu\right) \alpha_{\mathbf{q}}+g \alpha_{0}+q \sum_{\mathbf{k}} \alpha_{\mathbf{k q}} \\
\left(E-E_{0}\right) \alpha_{\mathbf{k q}} & =\left(\epsilon_{\mathbf{q}-\mathbf{k}, \mathrm{im}}+\epsilon_{\mathbf{k}}-\epsilon_{\mathbf{q}}\right) \alpha_{\mathbf{k q}}+g \alpha_{\mathbf{q}}+g \sum_{\mathbf{q}^{\prime}} \alpha_{\mathbf{k q q}^{\prime}} \\
\left(E-E_{0}\right) \alpha_{\mathbf{k} \mathbf{q}_{1} \mathbf{q}_{2}} & =\left(\epsilon_{\mathbf{q}_{1}+\mathbf{q}_{2}-\mathbf{k}, \mathrm{M}}+\epsilon_{\mathbf{k}}-\epsilon_{\mathbf{q}_{1}}-\epsilon_{\mathbf{q}_{2}}-\nu\right) \alpha_{\mathbf{k q}_{1} \mathbf{q}_{2}}+g \alpha_{\mathbf{k q} 1}-g \alpha_{\mathbf{k q} \mathbf{q}_{2}}+g \sum_{\mathbf{k}^{\prime}} \alpha_{\mathbf{k}^{\prime} \mathbf{k} \mathbf{q}_{1} \mathbf{q}_{2}} \\
\left(E-E_{0}\right) \alpha_{\mathbf{k}_{1} \mathbf{k}_{2} \mathbf{q}_{1} \mathbf{q}_{2}} & =\left(\epsilon_{\mathbf{q}_{1}+\mathbf{q}_{2}-\mathbf{k}_{1}-\mathbf{k}_{2}, \mathrm{im}}+\epsilon_{\mathbf{k}_{1}}+\epsilon_{\mathbf{k}_{2}}-\epsilon_{\mathbf{q}_{1}}-\epsilon_{\mathbf{q}_{2}}\right) \alpha_{\mathbf{k}_{1} \mathbf{k}_{2} \mathbf{q}_{1} \mathbf{q}_{2}}+g\left(\alpha_{\mathbf{k}_{2} \mathbf{q}_{1} \mathbf{q}_{2}}-\alpha_{\mathbf{k}_{1} \mathbf{q}_{1} \mathbf{q}_{2}}\right)
\end{aligned}
$$

where we have used $\alpha_{\mathbf{k} \mathbf{q}_{1} \mathbf{q}_{2}}=-\alpha_{\mathbf{k} \mathbf{q}_{2} \mathbf{q}_{1}}$ and $\alpha_{\mathbf{k}_{1} \mathbf{k}_{2} \mathbf{q}_{1} \mathbf{q}_{2}}=-\alpha_{\mathbf{k}_{2} \mathbf{k}_{1} \mathbf{q}_{1} \mathbf{q}_{2}}=-\alpha_{\mathbf{k}_{1} \mathbf{k}_{2} \mathbf{q}_{2} \mathbf{q}_{1}}$. For a static impurity, we have $\epsilon_{\mathbf{k}, \mathrm{im}}=\epsilon_{\mathbf{k}, \mathrm{M}}=0$, and thus the equations become independent of the angles between vectors.

[1] I. Bloch, J. Dalibard, and W. Zwerger, Rev. Mod. Phys. 80, 885 (2008).

[2] R. A. Barankov, L. S. Levitov, and B. Z. Spivak, Phys. Rev. Lett. 93, 160401 (2004).

[3] A. V. Andreev, V. Gurarie, and L. Radzihovsky, Phys. Rev. Lett. 93, 130402 (2004).

[4] M. H. Szymańska, B. D. Simons, and K. Burnett, Phys. Rev. Lett. 94, 170402 (2005).

[5] M. W. Zwierlein, A. Schirotzek, C. H. Schunck, and W. Ketterle, Science 311, 492 (2006).

[6] M. W. Zwierlein, A. Schirotzek, C. H. Schunck, and W. Ketterle, Nature (London) 442, 54 (2006).

[7] G. B. Partridge, W. Li, R. I. Kamar, Y. Liao, and R. G. Hulet, Science 311, 503 (2006).

[8] A. Schirotzek, C.-H. Wu, A. Sommer, and M. W. Zwierlein, Phys. Rev. Lett. 102, 230402 (2009).

[9] S. Nascimbène, N. Navon, K. J. Jiang, L. Tarruell, M. Teichmann, J. McKeever, F. Chevy, and C. Salomon, Phys. Rev. Lett. 103, 170402 (2009).
[10] Y.-a. Liao, A. S. C. Rittner, T. Paprotta, W. Li, G. B. Partridge, R. G. Hulet, S. K. Baur, and E. J. Mueller, Nature 467, 567 (2010).

[11] C. Kohstall, M. Zaccanti, M. Jag, A. Trenkwalder, P. Massignan, G. M. Bruun, F. Schreck, and R. Grimm, Nature (London) 485, 615 (2012).

[12] M. Koschorreck, D. Pertot, E. Vogt, B. Frölich, M. Feld, and M. Köhl, Nature (London) 485, 619 (2012).

[13] D. Mitra, P. T. Brown, P. Schauß, S. S. Kondov, and W. S. Bakr, ArXiv e-prints (2016), arXiv:1604.01479 [cond-mat.quant-gas].

[14] P. Nozières and C. T. de Dominicis, Phys. Rev. 178, 1097 (1969).

[15] M. Cetina, M. Jag, R. S. Lous, I. Fritsche, J. T. M. Walraven, R. Grimm, J. Levinsen, M. M. Parish, R. Schmidt, M. Knap, and E. Demler, "Ultrafast many-body interferometry of impurities coupled to a Fermi sea," ArXiv:1604.07423. 
[16] J. Goold, T. Fogarty, N. Lo Gullo, M. Paternostro, and T. Busch, Phys. Rev. A 85, 063632 (2011).

[17] M. Knap, A. Shashi, Y. Nishida, A. Imambekov, D. A. Abanin, and E. Demler, Phys. Rev. X 2, 041020 (2012).

[18] F. Chevy, Phys. Rev. A 74, 063628 (2006).

[19] N. Prokof'ev and B. Svistunov, Phys. Rev. B 77, 020408 (2008).

[20] J. Loschmidt, Sitzungsberichte der Akademie der Wissenschaften, Wien 73, 128 (1876).

[21] V. Gurarie and L. Radzihovsky, Annals of Physics 322, 2 (2007).

[22] D. S. Petrov, Phys. Rev. Lett. 93, 143201 (2004).

[23] A. D. McLachlan, Molecular Physics 8, 39 (1964).

[24] A. G. Basile and V. Elser, Phys. Rev. E 51, 5688 (1995).

[25] R. Combescot, A. Recati, C. Lobo, and F. Chevy, Phys. Rev. Lett. 98, 180402 (2007).

[26] R. Combescot and S. Giraud, Phys. Rev. Lett. 101, 050404 (2008).

[27] R. Combescot, S. Giraud, and X. Leyronas, Europhys. Lett. 88, 60007 (2009).

[28] M. Punk, P. T. Dumitrescu, and W. Zwerger, Phys. Rev. A 80, 053605 (2009).

[29] C. Mora and F. Chevy, Phys. Rev. A 80, 033607 (2009).

[30] C. J. M. Mathy, M. M. Parish, and D. A. Huse, Phys. Rev. Lett. 106, 166404 (2011).

[31] X. Cui and H. Zhai, Phys. Rev. A 81, 041602 (2010).

[32] Here we have assumed that all the closed channel molecules have been converted into spin-up atoms at the time of the measurement, which is reasonable if there is a sufficient delay after the second rf pulse.

[33] C. Trefzger and Y. Castin, Phys. Rev. A 85, 053612 (2012).
[34] O. Goulko, A. S. Mishchenko, N. Prokof'ev, and B. Svistunov, "Dark Continuum in the Spectral Function of the Resonant Fermi Polaron," ArXiv:1603.06963.

[35] P. Massignan, EPL (Europhysics Letters) 98, 10012 (2012).

[36] R. Schmidt and T. Enss, Phys. Rev. A 83, 063620 (2011).

[37] P. Massignan and G. M. Bruun, The European Physical Journal D 65, 83 (2011).

[38] G. D. Mahan, Many-particle physics, Physics of solids and liquids (Plenum, New York, NY, 1990).

[39] P. Massignan, Z. Yu, and G. M. Bruun, Phys. Rev. Lett. 110, 230401 (2013).

[40] J. Xu, Q. Gu, and E. J. Mueller, Phys. Rev. A 88, 023604 (2013).

[41] S. Tan, Ann. Phys. 323, 2952 (2008).

[42] O. I. Kartavtsev and A. V. Malykh, J. Phys. B 40, 1429 (2007).

[43] J. Levinsen, T. G. Tiecke, J. T. M. Walraven, and D. S. Petrov, Phys. Rev. Lett. 103, 153202 (2009).

[44] V. Efimov, Nucl. Phys. A 210, 157 (1973).

[45] Y. Castin, C. Mora, and L. Pricoupenko, Phys. Rev. Lett. 105, 223201 (2010).

[46] M. Cetina, M. Jag, R. S. Lous, J. T. M. Walraven, R. Grimm, R. S. Christensen, and G. M. Bruun, Phys. Rev. Lett. 115, 135302 (2015).

[47] A. Rosch and T. Kopp, Phys. Rev. Lett. 75, 1988 (1995).

[48] N. B. Jørgensen, L. Wacker, K. T. Skalmstang, M. M. Parish, J. Levinsen, R. S. Christensen, G. M. Bruun, and J. J. Arlt, Phys. Rev. Lett. 117, 055302 (2016).

[49] Y. Nishida, Phys. Rev. Lett. 114, 115302 (2015).

[50] W. Yi and X. Cui, Phys. Rev. A 92, 013620 (2015).

[51] P. Makotyn, C. E. Klauss, D. L. Goldberger, E. A. Cornell, and D. S. Jin, Nat Phys 10, 116 (2014). 